\title{
Nanotheranosties
}

2018; 2(3): 222-232. doi: 10.7150/ntno.25719

Research Paper

\section{Bio-transformation of Graphene Oxide in Lung Fluids Significantly Enhances Its Photothermal Efficacy}

\author{
Yun Liu1,2,\#, Yu Qi3,\#, Chunyang Yin ${ }^{2,4}$, Shunhao Wang2,4, Shuping Zhang5, An Xu' ${ }^{1}$, Wei Chen ${ }^{3 凶}$, Sijin \\ $\operatorname{Liu}^{2,4, 凶}$ \\ 1. Key Laboratory of High Magnetic Field and Ion Beam Physical Biology, Hefei Institutes of Physical Science, Chinese Academy of Sciences; Anhui Province \\ Key Laboratory of Environmental Toxicology and Pollution Control Technology, Hefei, Anhui 230031, China. \\ 2. State Key Laboratory of Environmental Chemistry and Ecotoxicology, Research Center for Eco-Environmental Sciences, Chinese Academy of Sciences, \\ Beijing 100085, China. \\ 3. College of Environmental Science and Engineering, Ministry of Education Key Laboratory of Pollution Processes and Environmental Criteria, Tianjin Key \\ Laboratory of Environmental Remediation and Pollution Control, Nankai University, Tianjin 300350, China. \\ 4. University of Chinese Academy of Sciences, Beijing 100049, China. \\ 5. Institute for Medical Engineering and Science, Massachusetts Institute of Technology, Cambridge, MA 02139, USA \\ \#These authors contributed equally to this work. \\ $\square$ Corresponding authors: E-mail: chenwei@nankai.edu.cn and sjliu@rcees.ac.cn
}

( $)$ Ivyspring International Publisher. This is an open access article distributed under the terms of the Creative Commons Attribution (CC BY-NC) license (https://creativecommons.org/licenses/by-nc/4.0/). See http://ivyspring.com/terms for full terms and conditions.

Received: 2018.03.01; Accepted: 2018.05.07; Published: 2018.05.20

\begin{abstract}
Rationale: Graphene oxide (GO) has shown great promises in biomedical applications, such as drug delivery and thermotherapeutics, owing to its extraordinary physicochemical properties. Nonetheless, current biomedical applications of GO materials are premised on the basis of predesigned functions, and little consideration has been given to the influence of bio-transformation in the physiological environment on the physicochemical properties and predesigned functionalities of these materials. Hence, it is crucial to uncover the possible influence on GO's physicochemical properties and predesigned functionalities for better applications.

Methods: Bio-transformed GOs were characterized by X-ray diffraction (XRD) spectra, Raman spectra, $X$-ray photoelectron spectroscopy (XPS) and Fourier transform infrared transmission (FT-IR) spectra. The morphologies of various GO materials were assessed via transmission electron microscopy (TEM), scanning electron microscopy (SEM) and atomic force microscopy (AFM) images. The photothermal (PTT) performance of different GO materials in vitro and in vivo were measured using $808 \mathrm{~nm}$ laser at a power density of $2 \mathrm{~W} / \mathrm{cm}^{2}$. The PTT efficacy was determined using transplanted 4T1 cells-derived breast tumors in mice.

Results: Bio-transformation of GO in the lung (a main target organ for GO to localize in vivo) can induce dramatic changes to its physicochemical properties and morphology, and consequently, its performances in biomedical applications. Specifically, GO underwent significant reduction in two simulated lung fluids, Gamble's solution and artificial lysosomal fluid (ALF), as evidenced by the increase of C/O ratio (the ratio of $\mathrm{C}$ content to $\mathrm{O}$ content) relative to pristine GO. Bio-transformation also altered GO's morphology, characterized by sheet folding and wrinkle formation. Intriguingly, bio-transformation elevated the PTT performance of GO in vitro, and this elevation further facilitated PTT-based tumor-killing efficacy in tumor cells in vitro and in a mouse model with transplanted tumors. Bio-transformation also compromised the interaction between drug with GO, leading to reduced drug adsorption, as tested using doxorubicin (DOX).

Conclusions: Transformation in Gamble's solution and ALF resulted in varied degrees of improved performances of GO, due to the differential effects on GO's physicochemical properties. Our findings unveiled an overlooked impact of GO bio-transformation, and unearthed a favorable trait of GO materials in thermotherapeutics and drug delivery in the lung microenvironment.
\end{abstract}

Key words: Graphene Oxide; Bio-transformation; Simulated Lung Fluids; Physicochemical Properties; Functionality 


\section{Introduction}

Since its discovery in 2004, graphene-based nanomaterials have been extensively studied in diverse biomedical applications [1-2]. Owing to its pronounced physicochemical properties, graphene oxide (GO) has been shown to be an excellent candidate for drug loading and cancer therapeutics (e.g. thermotherapeutics) [3-5]. Thus far, a number of approaches have been developed to modify GO physicochemical properties for better performance and various biomedical purposes, and the current progresses could be summarized into the following intertwined aspects: surface features, geometric parameters, physical states as a colloid and its surrounding protein coronas [6-7]. These studies provide the fundamental knowledge basis to understand the behaviors and physicochemical properties of nanomaterials on neighbor biomolecules and cells in the biological environments. However, it should be noted that all these strategies are premised on the basis of the predesigned physicochemical properties without considering the potential influences of transformation of GO materials in biological settings.

GO contains abundant reactive surface functional groups, and likely undergo significant alterations to the $3 S$ factors (size, shape and surface chemistry) once encountering ions and various biomolecules in the biological media [7-8]. Thus, after being administrated in vivo, transformation in biological media may greatly alter their predesigned properties, leading to loss of functional groups, oxidation and reduction of the materials, and subsequently either enhances or hinders their intended efficacies in biomedical applications [7, 9-10]. To date, little attention has been paid to the bio-transformation of GO materials in the biological environment, as well as the impacts on their biomedical performances.

It has been shown that GO materials may prove to be an ideal vehicle for lung-specific targeting. Primary cancers and metastatic cancers in the lung make up the largest number of cancer-related deaths, associated with tremendous morbidity and social and economic burden [11-14]. Selective lung targeting is intensively researched for cancer therapeutics. GO had been reported to target the lung through various exposure routes (e.g. intravenous (i.v.) administration, inhalation exposure) [15-19], being ascribed to the formation of GO-protein complexes that were readily and quickly caught by the abundant capillary vessels in the lung [10, 20-21]. Using a radiotracer approach, it was found that GO accumulated more quickly in the lung relative to other organs within a $1 \mathrm{~h}$ i.v. administration [10]. Strikingly, large-sized GO (1-5 $\mu \mathrm{m}$ in lateral dimension) was found to readily deposit in the lung 2 min post i.v. administration [20]. In a recent study, we found that $\mathrm{MoS}_{2} / \mathrm{GO}$ nanocomposites manifested selective lung targeting owing to GO's lung-targeting property, resulting in enhanced tumor killing efficacy [8]. The tendency of GO materials in targeting lung warrants a thorough understanding of their potential bio-transformation in the lung and the consequences, which have not been studied. Biotransformation is a generalized concept, defining the chemical alteration or transformation occurring within organisms or after the addition of chemical components in organisms [22-23]. In addition to investigation of biotransformation within organisms, researchers have explored the biotransformation process using body fluids, which are relatively simple and easy models to interpret the reaction processes and underlying mechanisms. For instance, due to formation of protein corona on GO, biotransformation in blood plasma greatly altered GO bio-reactivity towards cells [22].

In this study, we examined the bio-transformation of GO in two simulated lung fluids, Gamble's solution and artificial lysosomal fluid (ALF). The Gamble's solution mimics the interstitial fluid within the lung, and it is a mix of salts at $\mathrm{pH} 7.4$ [24], while ALF simulates the fluid after phagocytosis by alveolar and interstitial macrophages [25-26]. These two model solutions have been employed in investigating the alterations of various nanomaterials in physiological environments, including rare earth oxide nanoparticles [27], zinc oxide nanoparticles [28], carbon nanotubes [9], cobalt-based nanoparticles[29] and copper oxide nanoparticles [30]. We here endeavored to clarify the process that GO may undergo biotransformation in lung fluids with significant physicochemical properties alteration, which will subsequently elevate the functionality of GO in photothermal therapy (PTT) performance and drug delivery in the lungs. Our findings collectively underscore the importance of bio-transformation in altering the intrinsic physicochemical properties and morphology of GO materials, consequently affecting the efficacies of these materials for biomedical applications.

\section{Results and Discussion}

\section{Incubation in Lung Liquids Resulted in Significant GO Reduction and Functionality Alteration}

To investigate GO bio-transformation in the lung, we incubated GO in two simulated human lung fluids, Gamble's solution and ALF, for $5 \mathrm{~d}$ (the bio-transformed GO materials are referred to as 
"Gamble-GO" and "ALF-GO" hereafter). Treating GO with simulated lung fluids resulted in reduction of $\mathrm{GO}$, evidenced by the increase of the $\mathrm{C} / \mathrm{O}$ ratio, which is the ratio of $\mathrm{C}$ content to $\mathrm{O}$ content. The $\mathrm{X}$-ray diffraction (XRD) spectra (Figure 1A) revealed that GO underwent significant reduction upon incubation in the lung fluids, as the characteristic (002) peak of GO $\left(\sim 10^{\circ}\right)$ disappeared, whereas the intensity of the broad diffraction peak at $\sim 20^{\circ}$ increased for both Gamble-GO and ALF-GO in comparison to pristine GO. Consistently, the Raman spectra manifested an increase of the $I_{\mathrm{D}} / I_{\mathrm{G}}$ value from 0.61 for pristine GO to 0.82 and 0.84 for Gamble-GO and ALF-GO, respectively (Figure 1B), presumably attributable to the formation of new graphitic domains that are smaller in size than the ones present in pristine GO.

X-ray photoelectron spectroscopy (XPS) analysis showed the drastic alteration of GO surface functionality upon bio-transformation (Table 1, Figure S1). Specifically, the pristine GO contained $34.0 \%$ C-OH/C-O-C, much higher than Gamble-GO (29.0\%) and ALF-GO (26.0\%). Even more significant loss of carbonyl groups was observed, from $6.0 \%$ for GO to $1.9 \%$ for Gamble-GO and non-detectable for ALF-GO. In contrast, bio-transformation resulted in the increase in carboxyl groups, from $5.7 \%$ for GO to $8.7 \%$ for Gamble-GO and $26.0 \%$ for ALF-GO. The Fourier transform infrared transmission (FT-IR) spectra also revealed the loss of epoxy $\mathrm{C}-\mathrm{O}$ groups (bending band at $\sim 1217 \mathrm{~cm}^{-1}$ ) and carbonyl groups $(\mathrm{C}=\mathrm{O}$ stretching band at $\left.\sim 1670 \mathrm{~cm}^{-1}\right)$, but increase of hydroxyl groups (O-H bending band at $\sim 1385 \mathrm{~cm}^{-1}$ ) (Figure 1C).

\section{Bio-transformation of GO Caused Remarkable Morphological Changes}

Incubation in Gamble's solution and ALF also resulted in significant morphological changes of GO. Consistent with previous studies [16-17, 28, 31], The transmission electron microscopy (TEM) images showed that the pristine GO had the typical sheet-like morphology with even thickness and smooth surface (Figure 2A). By contrast, Gamble-GO and ALF-GO existed as thicker flakes with wrinkled and edge-crumpled structures (Figure 2B-C). Scanning electron microscopy (SEM) analysis also identified more wrinkled structures on Gamble-GO and ALF-GO than the pristine GO (Figure 2D-F). Consistent with the TEM and SEM data, the atomic force microscopy (AFM) (Figure 2G-I) images and statistical calculation data (more than 100 flakes for each material) (Figure 2J-L) showed that the thickness of GO nanosheets increased upon bio-transformation, particularly for Gamble-GO, likely due to the increase of van der Waals forces and hydrophobicity from reduction, resulting in greater tendency of nanosheets aggregation [9-10]. Intriguingly, between the two bio-transformed GOs, ALF-GO appeared to be less wrinkled than Gamble-GO (Figure 2), which might be ascribed to the high content of carboxyl groups of ALF-GO, leading to greater water dispensability.

\section{Bio-transformed GOs Exhibited Enhanced PTT Performance}

Owing to its extraordinary efficacy in photoelectron absorption and photothermal conversion, GO is considered to be an outstanding material for PTT performance $[5,32]$. With a bilayer hydrogel actuator driven by NIR irradiation, GO acts as an energy-converter during the PTT process [33]. GO administrated into tumors can convert the NIR laser into heat, leading to rapid elevation of the temperature in tumors and further the ablation of the tumor tissues [34]. For instance, PEGylated GO manifests an excellent in vivo photothermal conversion efficacy compared to carbon nanotubes [35]. Thereafter, the PTT performance for GO and bio-transformed GOs were compared in this study to illustrate the potential effects of GO biotransformation on its performances. Compared with pure water, pristine GO exhibited marked temperature elevation, with the highest temperature up to $49^{\circ} \mathrm{C}$ over $300 \mathrm{~s}$ irradiation at a power density of $2 \mathrm{~W} / \mathrm{cm}^{2}$ (Figure 3A). Both Gamble-GO and ALF-GO showed better PTT performance than pristine GO, with the highest temperature at nearly $55^{\circ} \mathrm{C}$ over 300 $\mathrm{s}$ irradiation (Figure 3A, $P<0.05$ ). To further evaluate the photothermal transduction capability of GO materials, pristine GO and bio-transformed GOs were irradiated by $808 \mathrm{~nm}$ laser at $2 \mathrm{~W} / \mathrm{cm}^{2}$ for $300 \mathrm{~s}$. Rapid cooling of the materials displayed typical photothermal profiles for these GOs (Figure 3B), demonstrating excellent thermal conductivity for these GOs. Furthermore, according to the data obtained from Figure 3B and S3, the time constant $\left(\tau_{s}\right)$ for the heat transfer was calculated to be $577 \mathrm{~s}$ for pristine GO, $368 \mathrm{~s}$ for Gamble-GO and $515 \mathrm{~s}$ for ALF-GO, respectively. It has been reported that the efficacy of PTT performance can be significantly affected by the physicochemical properties of GO. Specifically, due to the highly intact aromatic structure of graphene sheets, reduced GO (rGO), which is derived from reduction to partially restore the aromatic structure of graphene, possesses higher PTT efficacy than pristine GO [36-37]. Therefore, in our results, bio-transformed GOs, including Gamble-GO and ALF-GO, both exhibited superior PTT performance when comparing with pristine GO. These data thus highlighted the importance of tissue microenvironment in dictating GO's physicochemical properties and functionalities. 
Table 1. Physicochemical properties of pristine and bio-transformed GO materials.

\begin{tabular}{|c|c|c|c|c|c|c|c|c|c|c|c|}
\hline \multirow[b]{2}{*}{$\begin{array}{l}\text { GO/ Transformed } \\
\text { GOa }^{\text {a }}\end{array}$} & \multicolumn{4}{|l|}{$\mathrm{C}^{\mathrm{b}}(\mathrm{wt} \%)$} & \multirow[b]{2}{*}{$\begin{array}{l}\text { total } \mathrm{C}^{b} \\
(\mathrm{wt} \%)\end{array}$} & \multirow[b]{2}{*}{$\begin{array}{l}\text { total } \mathrm{O}^{b} \\
(\mathrm{wt} \%)\end{array}$} & \multirow[b]{2}{*}{$\begin{array}{l}\mathrm{C} / \mathrm{O} \\
\text { ratio }\end{array}$} & \multirow[b]{2}{*}{$\mathrm{pH}^{c}$} & \multirow[b]{2}{*}{$I_{D} / I_{G}$} & \multirow[b]{2}{*}{$D_{h^{d}}(\mathrm{~nm})$} & \multirow[b]{2}{*}{$\mathrm{PDI}^{d}$} \\
\hline & aromatic rings & $\begin{array}{l}\text { epoxy/ } \\
\text { hydroxyl }\end{array}$ & carbonyl & carboxyl & & & & & & & \\
\hline Pristine GO & 24.62 & 33.88 & 6.02 & 5.69 & 70.23 & 29.03 & 2.41 & 5.71 & 0.61 & $144.3 \pm 6.1$ & 0.277 \\
\hline Gamble-GO & 29.04 & 28.61 & 1.92 & 8.70 & 68.27 & 28.07 & 2.43 & 6.45 & 0.82 & $345.7 \pm 22.9$ & 0.508 \\
\hline ALF-GO & 22.88 & 26.19 & - & 25.19 & 74.98 & $23.58^{*}$ & 3.18 & 6.51 & 0.84 & $323.6 \pm 15.2$ & 0.459 \\
\hline
\end{tabular}

${ }^{a}$ Pristine GO represents untreated GO; Gamble-GO and ALF-GO represent GOs treated in Gamble's solution and ALF. ${ }^{b}$ Analyzed with X-ray photoelectron spectroscopy. ${ }^{c}$ $\mathrm{pH}$ value of $50 \mathrm{\mu g} / \mathrm{mL}$ GO/Gamble-GO/ALF-GO in deionized water. ${ }^{d} D_{\mathrm{h}}$ is the hydrodynamic diameter based on particle size distribution measured by DLS, and PDI is polydispersity index. ${ }^{*} \mathrm{P}<0.05$, compared to pristine GO.

A

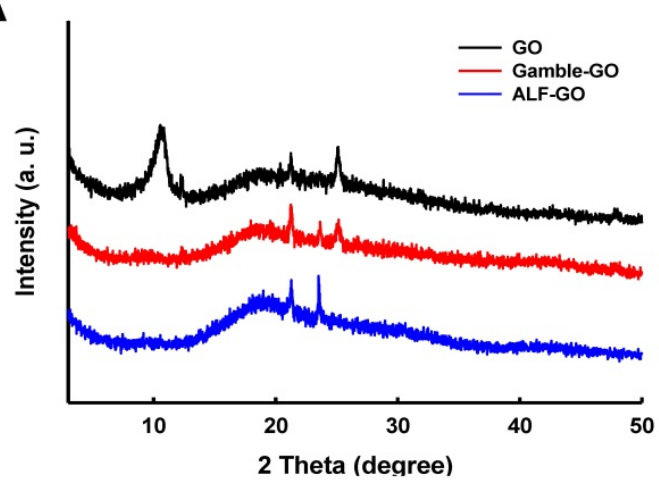

B

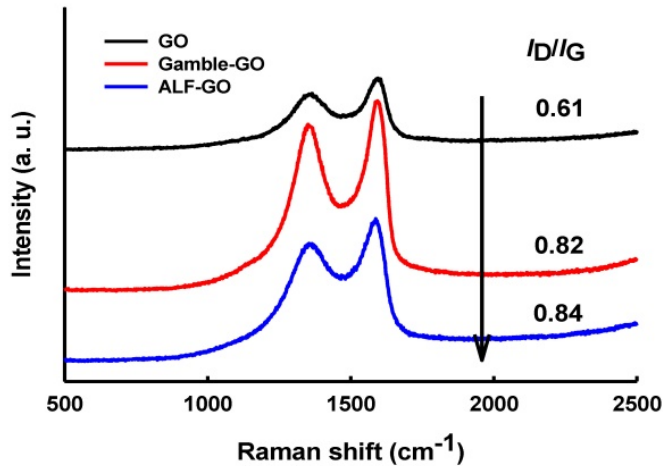

C

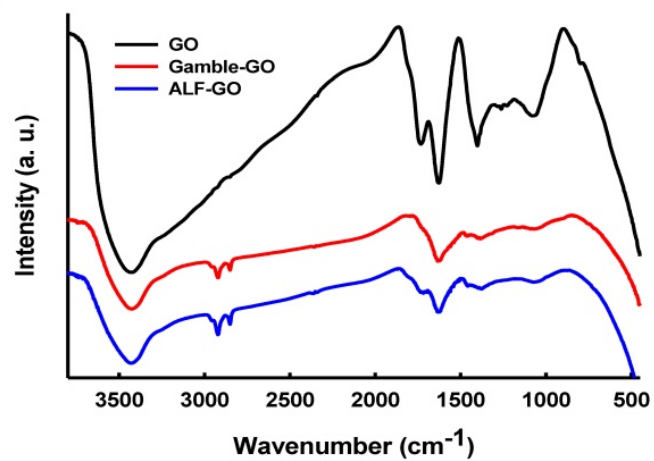

Figure 1. Physicochemical characterization of pristine GO, Gamble-GO and ALF-GO. (A) The XRD spectra, (B) Raman spectra and (C) FT-IR spectra of pristine and bio-transformed GO materials.

\section{ALF-GO revealed a greater PTT efficacy to repress tumor cells in vitro and in vivo}

Next, we examined the PTT efficacy of bio-transformed GOs alongside the pristine GO to kill tumors cells in vitro and in vivo. Since breast cancer is the most common cancer type in women and metastasis (heading to the lung or other sites) accounts for more than $90 \%$ of breast cancer-related deaths [38-39]. Therefore, 4T1 murine breast cancer cell line was used to assess the PTT performance of our materials. As shown in Figure S4, pristine GO showed mild dose-dependent photothermal cytotoxicity to $4 \mathrm{~T} 1$ cells at the power density of 2 $\mathrm{W} / \mathrm{cm}^{2}$ for $300 \mathrm{~s}$; by contrast, ALF-GO revealed remarkable photothermal cytotoxicity to $4 \mathrm{~T} 1$ cells in a dose-dependent manner $(P<0.05)$. Moreover, ALF-GO manifested superior PTT efficacy to pristine GO, as evidenced by $9 \%$ to $13 \%$ decline of viability in cells upon ALF-GO relative to that upon pristine GO at the concentrations from $25-100 \mu \mathrm{g} / \mathrm{mL}$ (Figure S4, $P$ $<0.05)$. These in vitro data demonstrated that bio-transformed ALF-GO harbored a greater efficacy than pristine GO in photothermal cytotoxicity towards cancer cells.

Furthermore, we first established a mouse model of orthotopic breast tumors through transplanting murine breast cancer 4T1 cells. PTT experiments were carried out through intra-tumor injection of ALF-GO in comparison to pristine GO (at $808 \mathrm{~nm}, 2 \mathrm{~W} / \mathrm{cm}^{2}$ for $300 \mathrm{~s}$, equals to $600 \mathrm{~J} / \mathrm{cm}^{2}$ as established previously [40]). Given that a similar PTT efficacy was found between ALF-GO and Gamble-GO in vitro, as described above, we here used ALF-GO in the animal experiment. Moreover, to clearly show the difference between ALF-GO and pristine GO in suppressing tumor growth due to differential PTT efficacy in-between, low-dose administration of materials (at $100 \mu \mathrm{g}$ for both ALF-GO and pristine GO) was employed. Figure 4A shows the temperature profiles over time post administration of the materials. After irradiation, tumor growth was closely monitored. As shown in Figure 4B and S5, tumor growth was remarkably restrained by more than $50 \%$ in ALF-GO-treated mice over the time course, compared to untreated mice and pristine GO-treated mice $(P<$ 0.05). By contrast, tumor growth in pristine GO-treated mice was not significantly altered relative to untreated mice, suggesting a low efficacy of PTT for pristine GO at the current dose in destroying tumor cells. Analogously, the final tumor size and tumor 
weight were markedly diminished in ALF-GO-treated mice compared to those in untreated mice and pristine GO-treated mice (Figure 4C and D). The health status, including the mortality and food intake, was normal for treated mice in this study. It is commonly believed that tumor tissue destruction will occur when temperature is higher than $43{ }^{\circ} \mathrm{C}$ [41-42]. As shown in Figure 4B, compared with the control group, there was a significant decrease of the tumor volume in GO-treated group on day 7; however, tumors increased to a comparable level in the control group at the end of the experiment, presumably resulting from the incomplete ablation of tumors and relapse. Overall, these results indicated that ALF-GO harbored a greater capability in PTT-based suppression on tumor development, pinpointing enhanced thermotherapeutic efficacy of GO materials after bio-transformation in the lung.

\section{Bio-transformation Compromised the Interaction between Drug with GO}

Ultrahigh surface area coupled with poly-aromatic surface structure makes GO suitable for efficient drug loading generally through п-п stacking, hydrophobic interaction and H-bonding [32, 43-45]. Therefore, in addition to the PTT efficacy, drug loading was evaluated for GO after bio-transformation in the lung fluids. Given that doxorubicin (DOX) is one of the mainstream chemotherapeutic drugs used in treating breast cancer [31, 43], we used it here as a model drug. Incubation in the lung fluids significantly reduced the drug loading capacity of GO (Figure 5), suggesting alleviated interaction between drug with GO after transformation. Specifically, at all three DOX concentrations tested, Gamble-GO and ALF-GO exhibited much lower loading capacity for DOX than the pristine GO. Furthermore, the differences between the pristine $\mathrm{GO}$ and the bio-transformed ones became larger with the increase of DOX concentration (Figure 5, $P<0.001$ ). For example, at a DOX concentration of $0.05 \mathrm{mg} / \mathrm{mL}$, the drug loading capacities of Gamble-GO and ALF-GO were $55 \%$ and $86 \%$, respectively, of that of the pristine $\mathrm{GO}$, whereas at a DOX concentration of $0.2 \mathrm{mg} / \mathrm{mL}$, the drug loading capacities of the bio-transformed ones reduced to only 47 and $61 \%$ of that of the pristine GO, respectively. It has been proposed that adsorption of DOX to GO is mainly driven by $\Pi-\Pi$ interaction [46-47] and H-bonding [45]. As the formation of new graphitic domains (showed in the increase of $\mathrm{I}_{D} / \mathrm{I}_{\mathrm{G}}$ ), the increase of $\Pi-\Pi$ interaction should increase the DOX loading capacity, while the decrease of O-functionalities upon GO reduction could significantly compromise the adsorptive interaction between GO and DOX via H-bonding. The stacking of GO sheets after transformation would largely reduce the available surface area for drug loading capacity. Even though ALF treatment resulted in more significant GO reduction, the drug-loading capacity of ALF-GO was less inhibited than Gamble-GO. This seems to be in line with the $\mathrm{H}$-bonding mechanism, in which ALF-GO contained greater amounts of surface carboxyl groups (an H-bonding moiety) than Gamble-GO (Table 1). Additionally, the DOX molecule contains ample $-\mathrm{NH}_{2}$ groups, allowing DOX to interact with the surface acidic groups on GO through Lewis acid-base interaction [48].

To achieve an effective therapeutic efficacy of targeted drug delivery, an ideal nano-vehicle in biomedical applications should possess two important traits: a) high affinity for loaded drug prior to arriving at the target therapeutic site; and b) effective release of loaded drug at the intended site. In this study, compromised interaction between DOX and GO upon bio-transformation in lung liquids may expedite drug release from $\mathrm{GO}$, which would enhance the therapeutic efficacy. This is a favorable trait of GO materials in drug delivery to the lung. Apart from PTT and drug delivery, GO displays promises in other aspects in cancer theranostics. For instance, GO-based materials have been developed for biosensing of tumor biomarkers [5], and multimodal imaging, including photoacoustic tomography (PAT) [49], positron emission tomography (PET) [50], magnetic resonance imaging (MRI) [51] and computer tomography (CT) [49]. In addition, multifunctional hybrid GO exhibits advantages in isolating and analyzing the circulating tumor cells [52].

In this study, we focused on studying the alterations of physicochemical properties and the inherent functionalities of GO materials upon bio-transformation. Hence, to clearly shed light on the molecular mechanism, only pristine GO with no surface modification was used here. With respect to the actual biomedical applications, it is necessary to optimize the property of GO with various approaches for surface modifications (e.g. modification with a tumor targeting motif), in order to achieve the multiple functionalization for therapeutics. In fact, the change on GO's surface properties will essentially alter its behaviors in biotransformation. However, to our knowledge, there is no research yet to look into biotransformation of modified GOs. Nonetheless, it is worth future investigation in this regard.

\section{Conclusions}

In conclusion, we here recognized remarkable changes of the physiochemical properties, morphology and resultantly functionalities for GO upon 
transformation in two simulated lung fluids: Gamble's solution and ALF. In fact, GO was reduced from the even sheet-like shape to a morphology with randomly wrinkled and stacked sheets, to differential extent for Gamble-GO and ALF-GO. Nonetheless, as a result of this bio-transformation, Gamble-GO and ALF-GO gained improved functionalities compared to pristine GO. Importantly, bio-transformed GO revealed greater PTT efficacy than pristine GO in killing tumor cells in vitro and in vivo. Meanwhile, bio-transformation in the lung fluids also compromis- ed the interaction between drug with GO. Only mild differences were defined between Gamble-GO and ALF-GO. These data together depicted a vital impact of GO's bio-transformation by lung fluids: improved PTT performance and presumably increased drug release, as delineated in Figure 6. This study thus unearthed the preferential feature of $\mathrm{GO}$ in thermotherapeutics and drug delivery for the lung targeting. This study may also offer more insights into the better design of nanomaterials for biomedical purposes in light of their bio-transformation.
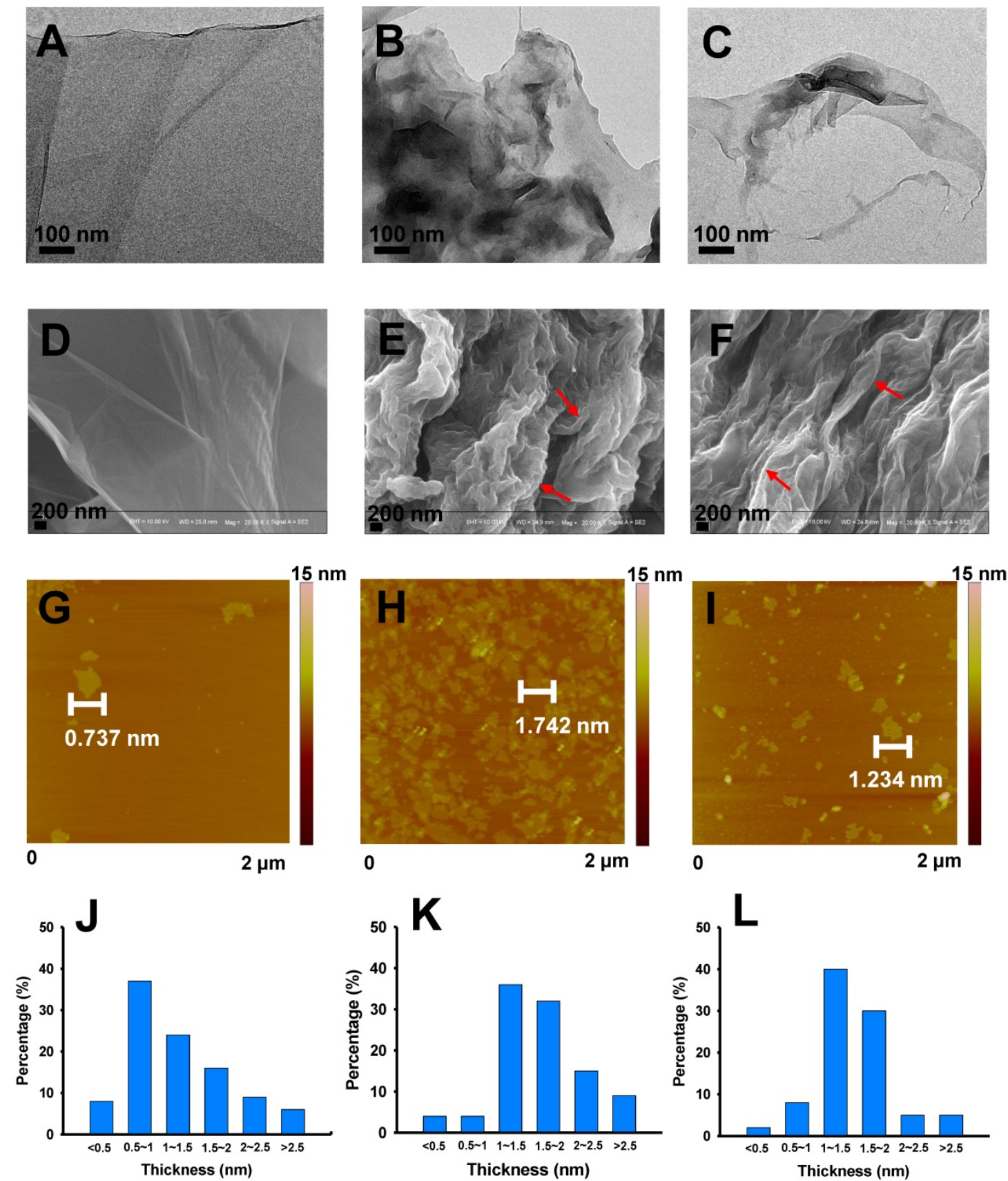

Figure 2. Morphology alteration of pristine and bio-transformed GO materials. Representative TEM, SEM and AFM images of (A, D, G) pristine GO, (B, E, H) Gamble-GO and (C, F, I) ALF-GO. Red arrows indicate the wrinkled and stacked sheets. The height profiles of (J) pristine GO, (K) Gamble-GO and (L) ALF-GO based on the AFM analysis. 


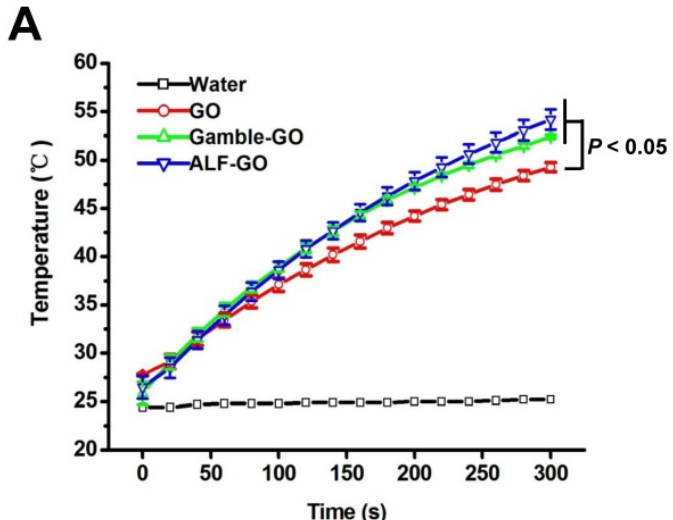

B

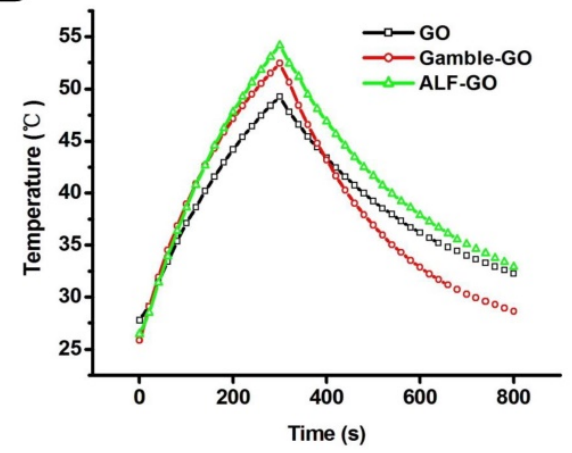

Figure 3. Photothermal profile of pristine $\mathbf{G O}$ and bio-transformed GOs. (A) PTT heating curves of pure water and various GO's solutions (at 0.8 $\mathrm{mg} / \mathrm{mL}$ ) under $808 \mathrm{~nm}$ laser irradiation $\left(2 \mathrm{~W} / \mathrm{cm}^{2}\right)$ for $5 \mathrm{~min}(n=3)$. (B) Temperature profile of GO solutions (at $0.8 \mathrm{mg} / \mathrm{mL}$ ) irradiated by $808 \mathrm{~nm}$ at a power density of $2 \mathrm{~W} / \mathrm{cm}^{2}$. The laser was turned off after irradiation for $300 \mathrm{~s}$.

\section{Materials and Methods}

\section{Preparation and characterization of GO materials.}

GO materials were purchased from the Nano Materials Tech Co. (China). Based on the information provided by the supplier, the product was synthesized using a modified Hummers method. Briefly, graphite powder and $\mathrm{KMnO}_{4}$ were dispersed in sequence into cooled $\mathrm{H}_{2} \mathrm{SO}_{4}\left(98 \%, 0{ }^{\circ} \mathrm{C}\right)$ while stirring vigorously. Then, the resultant suspension was continually stirred at $35^{\circ} \mathrm{C}$ for $3 \mathrm{~d}$. Afterward, distilled water was added and the suspension was kept at $98^{\circ} \mathrm{C}$ for $2 \mathrm{~h}$ and then cooled to $60^{\circ} \mathrm{C} . \mathrm{H}_{2} \mathrm{O}_{2}(30$ $\%)$ was added into the suspension to completely react with the excess $\mathrm{KMnO}_{4}$, and a bright yellow mixture was obtained. The solution was centrifuged and washed with $\mathrm{HCl}(30 \%)$ and distilled water until the $\mathrm{pH}$ value was neutral. All the inorganic and organic salts used to prepare simulated lung fluids were obtained from Sigma-Aldrich (China).

XRD spectra were recorded using a Rigaku D/Max III diffractometer (Rigaku Inc., Japan) with Cu K radiation, $\lambda=1.5418 \AA$. FT-IR spectra were obtained using a 110 Bruker TENSOR 27 apparatus (Bruker Optics Inc., Germany). Raman spectra were recorded with a Renishew inVia Raman spectrometer (RM2000, Renishew, U. K.). XPS (PHI 5000 VersaProbe, Ulvac-PHI, Japan) was employed for determining the surface elemental compositions of the nanomaterials. TEM (JEM-2100, JEOL, Japan), SEM (FEI Nanosem 430, FEI, USA) and AFM (Dimension 3100, DJ08, Veeco, USA) analysis were used for characterizing the physical dimensions of the nanomaterials.

\section{Transformation of $\mathbf{G O}$ in simulated lung fluids}

To examine the transformation of GO in the two synthesized lung fluids, a $25 \mu \mathrm{g} / \mathrm{mL}$ GO suspension was first prepared in Gamble's solution or ALF (the detailed recipes of these lung fluids are given in Table S1). Next, the suspension was slowly shaken (HWY-2122, China) at $80 \mathrm{rpm}$ at $37{ }^{\circ} \mathrm{C}$ for $5 \mathrm{~d}$, and then filtered through $0.22 \mu \mathrm{m}$ membrane filters. The bio-transformed GO materials retained on the filter was collected and washed by ultrasonication at $100 \mathrm{~W}$ for $30 \mathrm{~min}$. The rinsing procedure was repeated three times to remove extra salts. Afterwards, the bio-transformed GOs were collected by vacuum-drying and a fraction of the material was used for material characterizations. A stock solution $(1 \mathrm{mg} / \mathrm{mL})$ was prepared by sonicating in a water bath and was stored in borosilicate glass containers at $4^{\circ} \mathrm{C}$ in dark.

\section{PTT effect assessment}

The PTT performance of GO, Gamble-GO and ALF-GO in aqueous dispersion (at $0.8 \mathrm{mg} / \mathrm{mL}$, in 1 $\mathrm{mL}$ ) was measured using an $808 \mathrm{~nm}$ continuous wave (CW) GCSLS-05-007 semiconductor laser device (Daheng New Epoch Technology, Inc., Beijing, China) at a power density of $2 \mathrm{~W} / \mathrm{cm}^{2}$ for $5 \mathrm{~min}$. The temperature changes of the three samples were measured by a data logger thermometer (TES-1315, TES Electrical Electronic Corp.) every $20 \mathrm{~s}$.

\section{Methods for calculating the PTT time constants of pristine $\mathbf{G O}$ and bio-transformed GOs}

The calculation method for time constant $\left(\tau_{\mathrm{s}}\right)$ was described in previous reports [53-55], as follows. Briefly, during the photothermal heating process, the total energy balance for the system is expressed as:

$$
\sum_{i} m_{i} C_{P, i} \frac{d \Delta T}{d t}=Q_{N F}+Q_{S}-Q_{L O S S}
$$

Where $m(\mathrm{~g})$ denotes the mass of the solution $\left(m_{s}\right)$ and sample cuvette $\left(m_{q}\right), \mathrm{C}\left(\mathrm{J} /\left(\mathrm{g} \cdot{ }^{\circ} \mathrm{C}\right)\right)$ includes the constant-pressure heat capacity of solution $\left(c_{s}\right)$ and sample cuvette $\left(c_{q}\right), \Delta T\left({ }^{\circ} \mathrm{C}\right)$ is the difference between the solution temperature $T$ at time $t$ and the starting 
solution temperature $T_{0}, Q_{N F}(\mathrm{~mW})$ is calculated as the energy arising from the GO materials, and $Q_{\text {Loss }}(\mathrm{mW})$ is the lost thermal energy to the surrounding environment. In addition, $Q_{S}(\mathrm{~mW})$ is the energy input by the sample cuvette and the solvent (pure DI water).

$Q_{\text {Loss }}$ is linear with temperature for the outgoing thermal energy, which is expressed as:

$$
Q_{\text {LOSS }}=h S \Delta T=h S\left(T-T_{\text {Sur }}\right)
$$

Where $h\left(\mathrm{~mW} /\left(\mathrm{m}^{2}{ }^{\circ} \mathrm{C}\right)\right)$ is the heat transfer coefficient, $S\left(\mathrm{~m}^{2}\right)$ is the surface area of the container, $\Delta T$ is the temperature alteration that is defined as $T-T_{\text {sur }}, T\left({ }^{\circ} \mathrm{C}\right)$ is the water temperature and $T_{\text {sur }}\left({ }^{\circ} \mathrm{C}\right)$ is the environment temperature.

When the temperature rises at a maximum steady-state temperature $\mathrm{T}_{\operatorname{Max}}\left({ }^{\circ} \mathrm{C}\right)$, the system reaches the steady state. In order to solve $h S$, the following notation $\theta$ is used herein:

$$
\theta=\frac{T-T_{\text {Sur }}}{T_{\max }-T_{\text {Sur }}}
$$

A sample system time constant $\tau_{s}$ (s) is introduced:

$$
\tau_{s}=\frac{\sum_{i} m_{i} C_{P, i}}{h S}
$$

Substituting Equations (3) and (4) into Equation (1) and rearranging to obtain:

$$
\frac{d \theta}{d t}=\frac{1}{\tau_{S}}\left[\frac{Q_{N F}+Q_{S}}{h S\left(T_{\max }-T_{S u r}\right)}-\theta\right]
$$

At the cooling stage, the laser source is turned off, therefore the $\mathrm{Q}_{N F}+\mathrm{Q}_{S}=0$. Under this condition, Equation (5) becomes:

$$
d t=-\tau_{s} \frac{d \theta}{\theta}
$$

Note that after integration Equation (6), the Equation expresses as:

$$
t=-\tau_{s} \ln \theta
$$

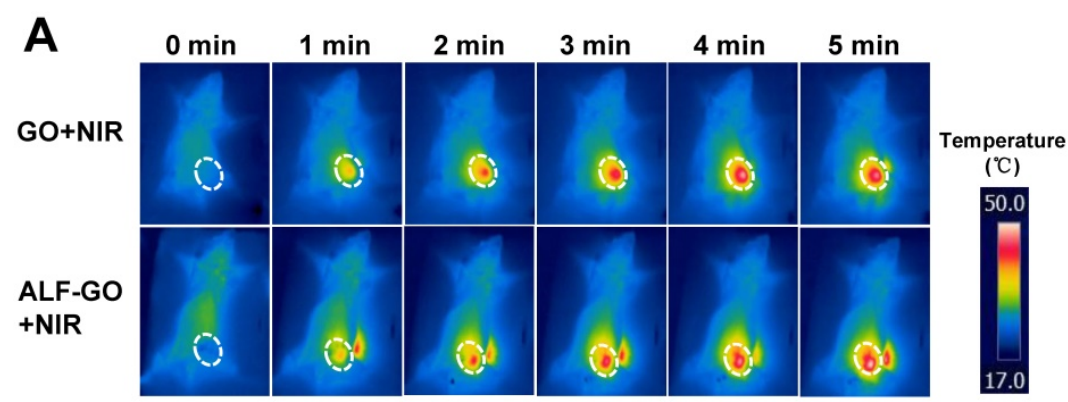

B
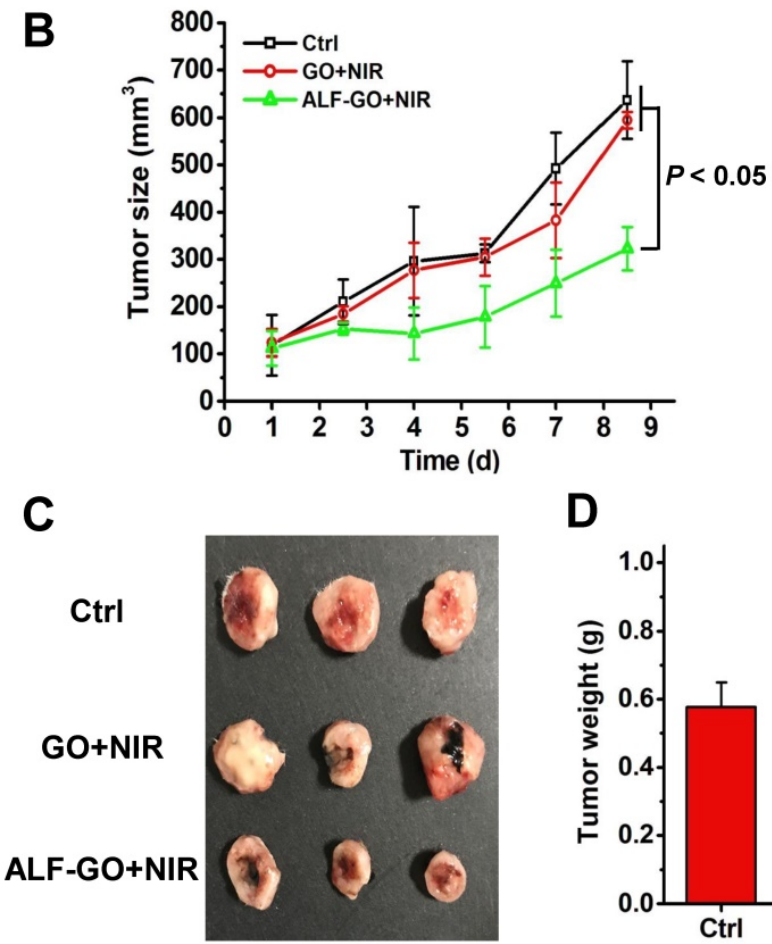

D

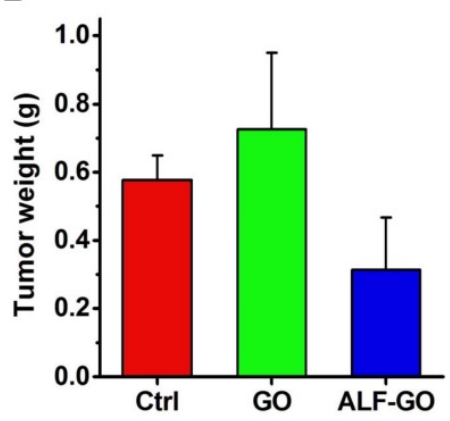

Figure 4. Photothermal suppression on tumor growth in vivo. (A) Temperature increase upon laser irradiation in implanted 4T1 tumors post administration of pristine GO and ALF-GO. Temperature profile was monitored over time post administration of materials. Dashed white circles delineate the tumor area in mice. (B) Tumor growth curves in mice upon PTT over the time course $(n=3)$. (C) Images of tumors after treatment. (D) The final tumor weight after treatment $(n=3)$. 


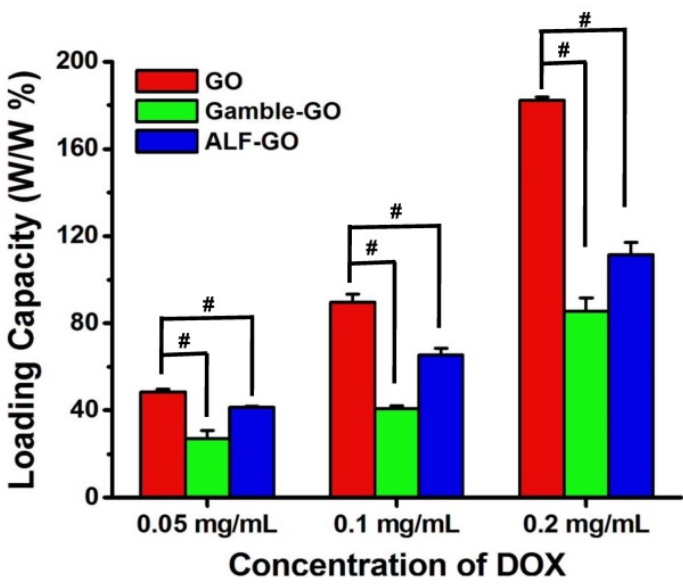

Figure 5. Drug loading capacity of GO, Gamble-GO and ALF-GO. The concentrations of all the nanomaterials used in this study were $0.1 \mathrm{mg} / \mathrm{mL}$ $(n=4)$. Pound sign (\#) denotes $P<0.001$, compared to pristine GO treated group.

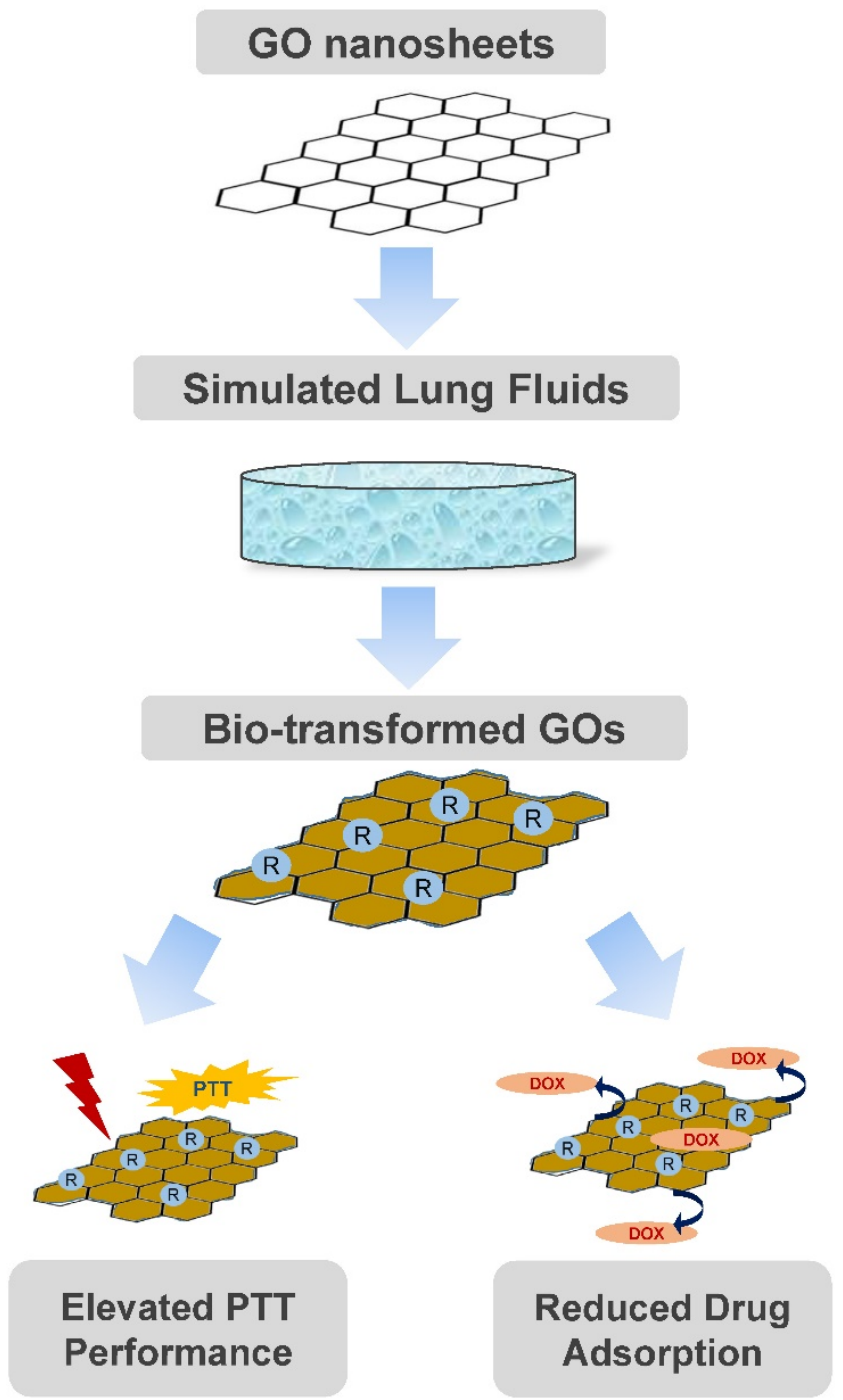

Figure 6. An overall schematic diagram depicting the morphology and functionality alterations of GO nanosheets after bio-transformation.

\section{Cell culture}

Mouse breast cancer cell line 4T1 was purchased from the Shanghai Cell Bank of Type Culture Collection of China. Cells were cultured continuously at $37{ }^{\circ} \mathrm{C}$ and $5 \% \mathrm{CO}_{2}$ in Dulbecco's modified Eagle medium (DMEM) (Gibco, USA) with 10\% fetal bovine serum (FBS), and 100 units/mL penicillin/ streptomycin (Invitrogen, USA).

\section{In vitro PTT efficacy of GO materials toward cancer cells}

Cells were first seeded at a density of $8 \times 10^{3}$ cells per well in 96-well plates and cultured for $24 \mathrm{~h}$. Cells were then treated with different concentrations (25 $\mu \mathrm{g} / \mathrm{mL}, 50 \mu \mathrm{g} / \mathrm{mL}, 100 \mu \mathrm{g} / \mathrm{mL}$ ) of pristine GO and ALF-GO for $1 \mathrm{~h}$. Afterwards, cells were treated with or without $808 \mathrm{~nm}$ laser at a power density of 2 $\mathrm{W} / \mathrm{cm}^{2}$ for $5 \mathrm{~min}$. After irradiation, cells were washed with PBS and incubated in fresh medium overnight. Cytotoxicity was determined using a cell counting kit (CCK)-8 (Solarbio, Beijing, China) following the manufacturer's instructions.

\section{Animal experimentation}

Six to seven-week-old female Balb/C mice (with body weight around $20 \mathrm{~g}$ ) were purchased from the Vital River Laboratories (Beijing, China). All mice were housed and used in accordance with the guidelines under the Animal Ethics Committee at the Research Center for Eco-Environmental Sciences, Chinese Academy of Sciences.

\section{In vivo examination of $\mathrm{PTT}$ efficacy in mice}

A mouse model with orthotopic breast tumor was first developed by injecting 5,000 4T1 cells into the fourth mammary fat pad (MFP) for each mouse, as described in our previous study [56]. When tumors reached a size about $100 \mathrm{~mm}^{3}$, mice bearing a similar size of tumors were randomly divided into three groups $(n=3)$. Treated mice were administrated through intra-tumor injection of GO and ALF-GO materials (with $100 \mu \mathrm{g}$ in $100 \mu \mathrm{L}$ ), respectively. Control mice received saline only. Tumors were then subjected to irradiation with NIR light $\left(2 \mathrm{~W} / \mathrm{cm}^{2}, 808\right.$ $\mathrm{nm})$ for $300 \mathrm{~s}$ right after the administration of materials. Thereafter, tumor growth was monitored every 1.5 days for 8.5 days. The tumor size was determined using the following formula: length $\times$ (width) ${ }^{2} / 2$. Finally, tumors were dissected and imaged when mice were sacrificed.

\section{Drug loading capacity}

The drug loading capacities of GO, Gamble-GO and ALF-GO were detected using ultraviolet and visible (UV-vis) spectrophotometer as reported [44]. 
Briefly, various GO nanomaterials $(0.1 \mathrm{mg} / \mathrm{mL})$ were mixed with free DOX at different concentrations $(0.05$, 0.1 and $0.2 \mathrm{mg} / \mathrm{mL}$ ) in PBS containing 2\% FBS (to avoid GO aggregation with salt ions). The mixtures were stirred overnight at room temperature. Unbounded DOX was washed away by centrifugation and repeatedly washed with PBS. All the supernatants obtained at each wash, were collected together and used to determine the amount of free DOX at the absorbance peak of $480 \mathrm{~nm}$. There was little difference in the UV-vis spectrum nearby the adsorption peak at $480 \mathrm{~nm}$ for these samples (Figure S6). Thus, $480 \mathrm{~nm}$ is a typical peak for DOX in UV-vis spectrum. This method in determining the DOX loading capacity on 2D nanomaterials was also used in previous studies $[8,44]$. DOX loading efficiency was calculated using the following equation:

$$
\text { Loading efficiency }(\%)=\frac{\mathrm{MV}\left(C_{0}-C_{e}\right)}{\mathrm{m}} \times 100 \%
$$

In the above equation, $\mathrm{M}(\mathrm{g} / \mathrm{mol})$ is the molar mass of DOX, V $(\mathrm{L})$ is the volume of supernatant, $C_{0}$ $(\mathrm{mol} / \mathrm{L})$ and $C_{\mathrm{e}}(\mathrm{mol} / \mathrm{L})$ are the initial and equilibrium concentrations of DOX in PBS buffer, respectively, and $\mathrm{m}(\mathrm{g})$ is the mass of GO nanomaterials.

\section{Statistical analysis}

All data were represented as the mean \pm standard deviation (SD). Statistical analysis was performed using independent $t$-test or one-way ANOVA test. $P$ value less than $0.05(P<0.05)$ was considered statistically significant.

\section{Acknowledgements}

This work was supported by the National "973" Program (Grant 2014CB932000), the Strategic Priority Research Program of the Chinese Academy of Sciences (Grant XDB14000000), and the National Natural Science Foundation of China (Grants 21425731, 21425729, 21637004 and 21607157).

\section{Supplementary Material}

Supplementary figures and tables. http://www.ntno.org/v02p0222s1.pdf

\section{Competing Interests}

The authors have declared that no competing interest exists.

\section{References}

1. Yang K, Feng L, Shi X, Liu Z. Nano-graphene in biomedicine: theranostic applications. Chem Soc Rev. 2013; 42: 530-47.

2. Shim G, Kim MG, Park JY, Oh YK. Graphene-based nanosheets for delivery of chemotherapeutics and biological drugs. Adv Drug Deliv Rev. 2016; 105: 205-27.
3. Sun X, Liu Z, Welsher $\mathrm{K}$, Robinson JT, Goodwin A, Zaric S, et al. Nano-graphene oxide for cellular imaging and drug delivery. Nano Res. 2008; 1: 203-12.

4. Zeng YP, Yang ZY, Li H, Hao YH, Liu C, Zhu L, et al. Multifunctional nanographene oxide for targeted gene-mediated thermochemotherapy of drug-resistant tumour. Sci Rep. 2017; 7: 43506.

5. Chen YJ, Wu YK, Sun BB, Liu SJ, Liu HY. Two-dimensional nanomaterials for cancer nanotheranostics. Small. 2017; 13: 1603446.

6. Papi M, Lauriola MC, Palmieri V, Ciasca G, Maulucci G, De Spirito M. Plasma protein corona reduces the haemolytic activity of graphene oxide nano and micro flakes. RSC Adv. 2015; 5: 81638-41.

7. Li YJ, Feng LZ, Shi XZ, Wang XJ, Yang YL, Yang K, et al. Surface coating-dependent cytotoxicity and degradation of graphene derivatives: towards the design of non-toxic, degradable nano-graphene. Small. 2014; 10 : 1544-54.

8. Liu Y, Peng J, Wang SH, Xu M, Gao M, Xia T, et al. Molybdenum disulfide/graphene oxide nanocomposites show favorable lung targeting and enhanced drug loading/tumor-killing efficacy with improved biocompatibility. NPG Asia Mater. 2018; 10: e458.

9. Osmond-McLeod MJ, Poland CA, Murphy F, Waddington L, Morris H, Hawkins SC, et al. Durability and inflammogenic impact of carbon nanotubes compared with asbestos fibres. Part Fibre Toxicol. 2011; 8: 15.

10. Zhang XY, Yin JL, Peng C, Hu WQ, Zhu ZY, Li WX, et al. Distribution and biocompatibility studies of graphene oxide in mice after intravenous administration. Carbon. 2011; 49: 986-95.

11. Siegel RL, Miller KD, Jemal A. Cancer statistics. CA Cancer J Clin. 2016; 66: 7-30.

12. Torre LA, Siegel RL, Jemal A. Lung Cancer Statistics. Adv Exp Med Biol. 2016; 893: 1-19.

13. Massague J, Obenauf AC. Metastatic colonization by circulating tumour cells. Nature. 2016; 529: 298-306.

14. Lambert AW, Pattabiraman DR, Weinberg RA. Emerging biological principles of metastasis. Cell. 2017; 168: 670-91.

15. Sanchez VC, Jachak A, Hurt RH, Kane AB. Biological interactions of graphene-family nanomaterials: an interdisciplinary review. Chem Res Toxicol. 2012; 25: 15-34

16. Ma J, Liu R, Wang X, Liu Q, Chen YN, Valle RP, et al. Crucial role of lateral size for graphene oxide in activating macrophages and stimulating pro-inflammatory responses in cells and animals. ACS Nano. 2015; 9: 10498-515.

17. Xu M, Zhu JQ, Wang FF, Xiong YJ, Wu YK, Wang QQ, et al. Improved in vitro and in vivo biocompatibility of graphene oxide through surface modification: Poly(Acrylic Acid)-functionalization is superior to PEGylation. ACS Nano. 2016; 10: 3267-81.

18. Chen SM, Xiong CO, Liu HH, Wan $\mathrm{OQ}$, Hou J, He Q, et al. Mass spectrometry imaging reveals the sub-organ distribution of carbon nanomaterials. Nat Nanotechnol. 2015; 10: 176-82.

19. Nurunnabi M, Khatun Z, Huh KM, Park SY, Lee DY, Cho KJ, et al. In vivo biodistribution and toxicology of carboxylated graphene quantum dots. ACS Nano. 2013; 7: 6858-67.

20. Liu JH, Yang ST, Wang H, Chang Y, Cao A, Liu Y. Effect of size and dose on the biodistribution of graphene oxide in mice. Nanomedicine (Lond). 2012; 7: 1801-12.

21. Wen KP, Chen YC, Chuang CH, Chang HY, Lee CY, Tai NH. Accumulation and toxicity of intravenously-injected functionalized graphene oxide in mice. J Appl Toxicol. 2015; 35: 1211-8.

22. Hu XG, Li DD, Mu L. Biotransformation of graphene oxide nanosheets in blood plasma affects their interactions with cells. Environ Sci-Nano. 2017; 4: 1569-78.

23. Chouhan RS, Qureshi A, Yagci B, Gülgün MA, Ozguz V, Niazi JH. Biotransformation of multi-walled carbon nanotubes mediated by nanomaterial resistant soil bacteria. Chem Eng J. 2016; 298: 1-9.

24. Marques MRC, Loebenberg R, Almukainzi M. Simulated biological fluids with possible application in dissolution testing. Dissolut Technol. 2011; 18: 15-28.

25. Colombo C, Monhemius AJ, Plant JA. Platinum, palladium and rhodium release from vehicle exhaust catalysts and road dust exposed to simulated lung fluids. Ecotoxicol Environ Saf. 2008; 71: 722-30.

26. Hedberg Y, Gustafsson J, Karlsson HL, Moller L, Odnevall Wallinder I. Bioaccessibility, bioavailability and toxicity of commercially relevant iron- and chromium-based particles: in vitro studies with an inhalation perspective. Part Fibre Toxicol. 2010; 7: 23.

27. Li $\mathrm{R}$, Ji Z Chang $\mathrm{CH}$, Dunphy DR, Cai X, Meng $\mathrm{H}$, et al Surface interactions with compartmentalized cellular phosphates explain rare earth oxide nanoparticle hazard and provide opportunities for safer design. ACS Nano. 2014; 8: 1771-83.

28. Adamcakova-Dodd A, Stebounova LV, Kim JS, Vorrink SU, Ault AP, O'Shaughnessy PT, et al. Toxicity assessment of zinc oxide nanoparticles using sub-acute and sub-chronic murine inhalation models. Part Fibre Toxicol. 2014; 11: 15.

29. Jeong J, Han Y, Poland CA, Cho WS. Response-metrics for acute lung inflammation pattern by cobalt-based nanoparticles. Part Fibre Toxicol. 2015; 12: 13 .

30. Semisch A, Ohle J, Witt B, Hartwig A. Cytotoxicity and genotoxicity of nano and microparticulate copper oxide: role of solubility and intracellular bioavailability. Part Fibre Toxicol. 2014; 11: 10 
31. Zhu JQ, Xu M, Gao M, Zhang ZH, Xu Y, Xia T, et al. Graphene oxide induced perturbation to plasma membrane and cytoskeletal meshwork sensitize cancer cells to chemotherapeutic agents. ACS Nano. 2017; 11: 2637-51.

32. Tian B, Wang C, Zhang S, Feng LZ, Liu Z. Photothermally enhanced photodynamic therapy delivered by nano-graphene oxide. ACS Nano. 2011; 5 : 7000-9.

33. Huang YY, Chen CY, Li HT, Xiao AX, Guo T, Guan BO. Insight into the local near-infrared photothermal dynamics of graphene oxide functionalized polymers through optical microfibers. Phys Chem Chem Phys. 2018; 20: 5256-63.

34. Wang SG, Li K, Chen Y, Chen HR, Ma M, Feng JW, et al. Biocompatible PEGylated MoS2 nanosheets: controllable bottom-up synthesis and highly efficient photothermal regression of tumor. Biomaterials. 2015; 39: 206-17.

35. Robinson JT, Welsher K, Tabakman SM, Sherlock SP, Wang H, Luong R, et al. High performance in vivo Near-IR $(>1$ mum) imaging and photothermal cancer therapy with carbon nanotubes. Nano Res. 2010; 3: 779-93.

36. Robinson JT, Tabakman SM, Liang Y, Wang H, Casalongue HS, Vinh D, et al. Ultrasmall reduced graphene oxide with high near-infrared absorbance for photothermal therapy. J Am Chem Soc. 2011; 133: 6825-31.

37. Chen YW, Su YL, Hu SH, Chen SY. Functionalized graphene nanocomposites for enhancing photothermal therapy in tumor treatment. Adv Drug Deliv Rev. 2016; 105: 190-204.

38. Liu S, Goldstein RH, Scepansky EM, Rosenblatt M. Inhibition of rho-associated kinase signaling prevents breast cancer metastasis to human bone. Cancer Res. 2009; 69: 8742-51.

39. Saha M, Kumar S, Bukhari S, Balaji SA, Kumar P, Hindupur SK, et al. AMPK-AKT double negative feedback loop in breast cancer cells regulates their adaptation to matrix deprivation. Cancer Res. 2018.

40. Virani NA, Davis C, McKernan P, Hauser P, Hurst RE, Slaton J, et al. Phosphatidylserine targeted single-walled carbon nanotubes for photothermal ablation of bladder cancer. Nanotechnology. 2018; 29: 035101.

41. O'Neal DP, Hirsch LR, Halas NJ, Payne JD, West JL. Photo-thermal tumor ablation in mice using near infrared-absorbing nanoparticles. Cancer Lett. 2004; 209: 171-6.

42. Dickerson EB, Dreaden EC, Huang X, El-Sayed IH, Chu H, Pushpanketh S, et al. Gold nanorod assisted near-infrared plasmonic photothermal therapy (PPTT) of squamous cell carcinoma in mice. Cancer Lett. 2008; 269: 57-66.

43. Liu T, Wang C, Gu X, Gong H, Cheng L, Shi XZ, et al. Drug Delivery with PEGylated MoS2 Nano-sheets for Combined Photothermal and Chemotherapy of Cancer. Adv Mater. 2014; 26: 3433-40.

44. Yin WY, Yan L, Yu J, Tian G, Zhou LJ, Zheng XP, et al. High-throughput synthesis of single-layer MoS2 nanosheets as a Near-Infrared photothermal-triggered drug delivery for effective cancer therapy. ACS Nano. 2014; 8: 6922-33.

45. Yang XY, Zhang XY, Liu ZF, Ma YF, Huang Y, Chen Y. High-efficiency loading and controlled release of doxorubicin hydrochloride on graphene oxide. J Phys Chem C. 2008; 112: 17554-58.

46. Zhang LM, Xia JG, Zhao QH, Liu LW, Zhang ZJ. Functional graphene oxide as a nanocarrier for controlled loading and targeted delivery of mixed anticancer drugs. Small. 2010; 6: 537-44.

47. Liu Z, Sun XM, Nakayama-Ratchford N, Dai HJ. Supramolecular chemistry on water-soluble carbon nanotubes for drug loading and delivery. ACS Nano. 2007; 1: 50-56.

48. Hou L, Zhu DQ, Wang XM, Wang LL, Zhang CD, Chen W. Adsorption of phenanthrene, 2-naphthol, and 1-naphthylamine to colloidal oxidized multiwalled carbon nanotubes: effects of humic acid and surfactant modification. Environ Toxicol Chem. 2013; 32: 493-500.

49. Zhang $Y X$, Zhang $\mathrm{H}$, Wang $\mathrm{YK}$, Wu HX, Zeng B, Zhang $\mathrm{YJ}$, et al. Hydrophilic graphene oxide/bismuth selenide nanocomposites for CT imaging, photoacoustic imaging, and photothermal therapy. J Mater Chem B. 2017; 5: 1846-55.

50. Jang SC, Kang SM, Lee JY, Oh SY, Vilian AE, Lee I, et al. Nano-graphene oxide composite for in vivo imaging. Int J Nanomedicine. 2018; 13: 221-34.

51. Tao YG, Zhu LG, Zhao YY, Yi X, Zhu LB, Ge F, et al. Nano-graphene oxide-manganese dioxide nanocomposites for overcoming tumor hypoxia and enhancing cancer radioisotope therapy. Nanoscale. 2018; 10: 5114-23.

52. Pramani KA, Jones S, Gao Y, Sweet C, Vangara A, Begum S, et al. Multifunctional hybrid graphene oxide for circulating tumor cell isolation and analysis. Adv Drug Deliv Rev. 2018.

53. Feng W, Chen L, Qin M, Zhou XJ, Zhang QQ, Miao YK, et al. Flower-like PEGylated MoS2 nanoflakes for near-infrared photothermal cancer therapy. Sci Rep. 2015; 5: 17422 .

54. Roper DK, Ahn W, Hoepfner M. Microscale heat transfer transduced by surface plasmon resonant gold nanoparticles. J Phys Chem C. 2007; 111: 3636-41.

55. Feng $W$, Zhou $X J$, Nie $W$, Chen $L$, Qiu $K X$, Zhang $Y Z$, et al $\mathrm{Au} /$ polypyrrole@Fe3O4 nanocomposites for MR/CT dual-modal imaging guided-photothermal therapy: an in vitro study. ACS Appl Mater Interfaces. 2015; 7: 4354-67.

56. Gao J, Sanchez-Purra M, Huang H, Wang SH, Chen YN, Yu XF et al. Synthesis of different-sized gold nanostars for Raman bioimaging and photothermal therapy in cancer nanotheranostics. Sci China Chem. 2017; 60: 1219-29. 